\title{
Estudo do Acoplamento Poço-Reservatório com Fluidodinâmica Computacional
}

\author{
R. T. MARQUES, A. R. de MELO, T. S. KLEIN, R. A. MEDRONHO \\ Universidade Federal do Rio de Janeiro, Departamento de Engenharia Química \\ E-mail para contato: ric_terra@yahoo.com.br
}

RESUMO - Nas duas últimas décadas, poços horizontais foram empregados em larga escala nas perfurações off-shore brasileiras. Frequentemente, em simulações de poços, é adotada a hipótese de condutividade infinita, isto é, assume-se como desprezível a perda de carga sofrida devido ao escoamento no interior do poço horizontal.

Esta abordagem, em médio prazo, pode trazer aspectos adversos à exploração, como por exemplo, a formação de cone de água ou gás, caracterizado pela produção antecipada dessas fases.

Este trabalho realizou, através de fluidodinâmica computacional, um estudo de caso, visando investigar a influência das hipóteses de condutividade finita e infinita, assim como a variação da rugosidade relativa e do comprimento do poço. Para isso, fez-se um acoplamento poço-reservatório 3D-1D, o qual mostrou-se como uma metodologia adequada, uma vez que é capaz de descrever com boa precisão a drenagem de um reservatório.

Os resultados indicaram que a hipótese de condutividade infinita não é válida para o caso estudado, e os resultados obtidos considerando-se condutividade finita apresentaram grande similaridade com os dados experimentais da literatura, revelando que a perda de carga foi responsável por uma queda em 30\% na produção. Foi demonstrado também que aumentar o comprimento do poço não compensaria economicamente.

Também foi possível compreender a causa de maior ocorrência de cones de água e gás na região próxima ao calcanhar (heel), devido ao maior gradiente de pressão.

\section{INTRODUÇÃO}

Nas duas últimas décadas, poços horizontais e direcionais foram empregados em larga escala nas perfurações offshore brasileiras. Os poços horizontais possuem uma série de vantagens quando comparados a outros tipos de poços, tais como: maior área efetiva de drenagem, maior capacidade de explotação em reservatórios delgados, fraturas naturais, formações com baixa permeabilidade, redução na formação de cones de água/gás, e aumento da eficiência na recuperação secundária.

Diversas metodologias foram propostas ao longo dos anos a fim de se obter a integração poçoreservatório, dentre elas destacam-se dois modelos conhecidos como hipótese da condutividade finita e infinita. Estes modelos se diferenciam pelo fato de que enquanto na condutividade infinita a perda 
de carga ao longo do poço é desprezada, na outra abordagem, a perda de carga é levada em consideração (Joshi, 1991).

A literatura revela que, frequentemente em simulações de reservatórios, é adotada a hipótese de condutividade infinita. Autores como Ozkan et al. (1999) discutem que essa simplificação pode ser aplicada restritamente a sistemas de baixa produção, em que o gradiente de pressão devido ao escoamento através do poço é desprezível em relação à perda de carga no reservatório (drawdown), enquanto que autores como Dickstein et al. (1997) indicam ausência de simetria na distribuição de vazão ao longo de poços horizontais quando a perda de carga é considerada.

Sasoni et al. (2007) estudaram um acoplamento poço-reservatório utilizando ferramentas de fluidodinâmica computacional (CFD) para ambas abordagens, infinita e finita, por meio da interação entre as simulações 3D do reservatório e a simulação 3D do poço. Isto permitiu a obtenção da solução completa do escoamento interno assim como o cálculo da perda de carga sem que fossem utilizadas correlações empíricas. O custo computacional foi, entretanto, significativo. Essa abordagem pode ser caracterizada como 3D-3D.

\section{OBJETIVOS}

O objetivo principal deste trabalho é investigar a fluidodinâmica do escoamento na interface poço-reservatório para se avaliar de que forma as hipóteses de condutividade finita e infinita influenciam na produtividade estimada. Foi realizado um estudo de caso de um poço horizontal por meio de fluidodinâmica computacional (CFD), sendo proposta uma simulação 3D-1D do acoplamento, isto é, simular o reservatório em 3D e obter a perda de carga no poço por meio de correlação empírica calculada em 1D. Desta forma, espera-se reduzir o esforço computacional e, consequentemente, reduzir o tempo de simulação. Por fim, busca-se analisar os perfis de produção do poço horizontal submetidos a diferentes especificações de projeto.

\section{METODOLOGIA}

O presente trabalho está focado na simulação com CFD de um reservatório que é explorado por um poço horizontal de modo que os sistemas de produção estejam integrados de forma dinâmica. Desta forma, o acoplamento poço-reservatório é considerado na modelagem do problema.

Ao invés de modelar o reservatório por meio de correlações empíricas, como feito por Ozkan et al. (1993), será proposta a resolução das equações de conservação de massa e momento, através da discretização do domínio e aplicação do método de volumes finitos por meio da utilização do software comercial de fluidodinâmica computacional ANSYS FLUENT 13.0.

A geometria primária foi baseada no modelo simples de paralelepípedo representando o reservatório, e um cilindro representando o poço. Entretanto, por questões de simplificação, a geometria foi reduzida a um quarto da forma original, explorando suas simetrias, reduzindo-se o reservatório e o poço a uma seção de um quarto de paralelepípedo e cilindro, respectivamente. 
Sendo assim, foi gerada uma geometria na forma de um paralelepípedo com as dimensões $2500 \mathrm{x}$ 850 x 11 metros. Na Tabela 1, podem ser encontradas as dimensões do reservatório e do poço, bem como as propriedades físicas do óleo utilizado nas simulações.

\begin{tabular}{cc} 
Tabela 1 - Dados do reservatório, poço horizontal e óleo. \\
\hline Parâmetro & Valor \\
\hline Raio de Drenagem - rd (m) & 850 \\
Espessura do Reservatório (m) & 11 \\
Porosidade (\%) & 25 \\
Permeabilidade Horizontal (mD) & 8500 \\
Permeabilidade Vertical (mD) & 1500 \\
Drawdown (bar) & 2,02 \\
Comprimento do trecho horizontal do poço & 800 \\
(m) & 0,1524 \\
Diâmetro do Poço (m) & 881 \\
Massa Específica do Óleo (kg/m³) & 1,43 \\
Viscosidade Dinâmica do óleo (cP) &
\end{tabular}

Uma malha hexaédrica estruturada contendo em torno de 3,1 milhões de elementos foi identificada como independente e utilizada para discretizar o domínio. O reservatório recebeu um tratamento em multiblocos vertical e horizontal para facilitar o refino e torná-lo mais localizado, como ilustrado na Figura 1. Pode-se notar o refinamento da malha nas proximidades da interface poço-reservatório.

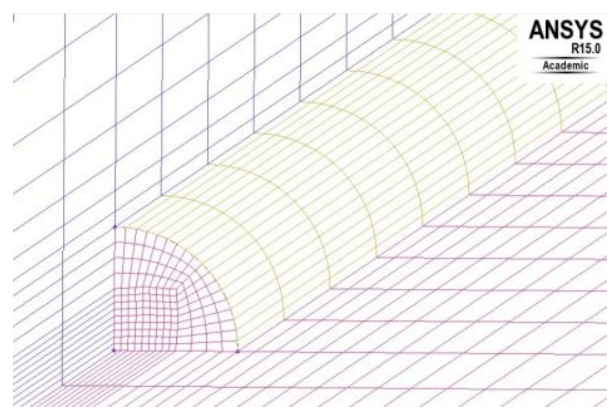

Figura 1 - Detalhe do refinamento da malha nas proximidades do poço.

O reservatório foi modelado em três dimensões como um meio poroso anisotrópico, descrito pela Lei de Darcy, com valores constantes para a permeabilidade vertical, a permeabilidade horizontal e a porosidade, como indicado na Tabela 1 . O escoamento no interior do reservatório foi aproximado para monofásico (óleo somente), contendo baixo número de Reynolds (laminar), incompressível, isotérmico e simulado em estado estacionário. 
O poço horizontal foi resolvido analiticamente, como $1 \mathrm{D}$, por equações previamente estabelecidas para o modelo, de forma que fosse possível realizar um cálculo sequencial. $\mathrm{O}$ modelo proposto tem início pela resolução das equações de conservação no reservatório empregando-se o simulador ANSYS FLUENT 13.0. A vazão mássica do reservatório para o poço é exportada para uma rotina de programação e sobre ela são realizados cálculos para se obter a perda de carga no poço, que em seguida é "realimentada" no código numérico para a simulação com CFD. O simulador resolve novamente as equações de conservação para o novo perfil de pressão do poço, obtendo um valor atualizado de fluxo mássico para o poço horizontal, este processo permanece num loop iterativo até que o critério de convergência de $10^{-6}$ seja obtido com relação ao balanço de massa. De forma análoga ao reservatório, o escoamento no interior do poço foi considerado monofásico, isotérmico e incompressível. Logo, se trata de um acoplamento explícito, como exibido na Figura 2, que apresenta um fluxograma da sequência de cálculo.

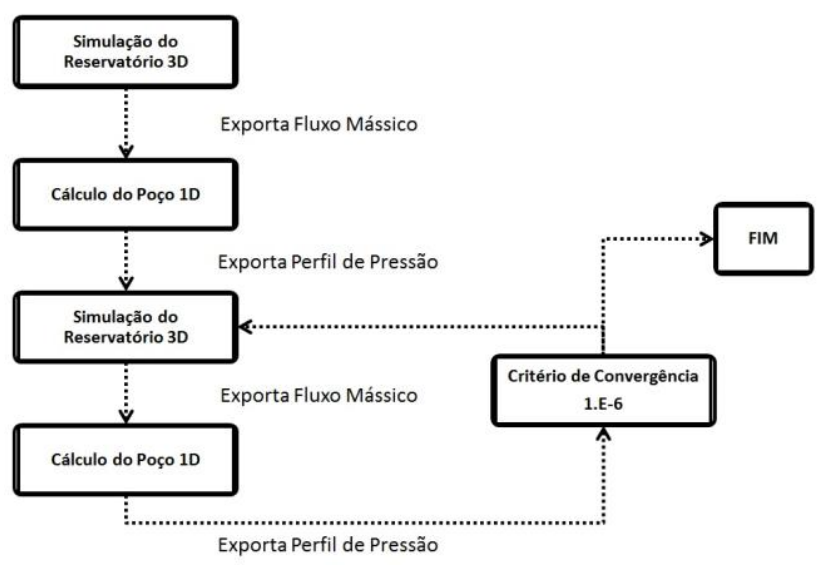

Figura 2 - Fluxograma do acoplamento poço-reservatório.

O equacionamento utilizado tem como objetivo montar uma estratégia de cálculo sequencial que, a partir da vazão mássica fornecida pelo simulador, obtenha a perda de carga ao longo do poço horizontal.

$Q_{w i}=Q_{R i}+Q_{w i-1}$

$v_{i}=\frac{Q_{w i}}{A}$

$\operatorname{Re}_{i}=\frac{\rho v_{i} D}{\mu}$ 


$$
\begin{aligned}
& f_{i}=\left\{\left(\frac{64}{\operatorname{Re}_{i}}\right)^{8}+9,5\left[\ln \left[\left(\frac{\varepsilon}{3,7 D}\right)+5,74 \mathrm{Re}_{i}^{-0,9}\right]-\left(\frac{2500}{\operatorname{Re}_{i}}\right)^{6}\right]^{-16}\right\}^{0,125} \\
& \Delta P_{i}=-\left(\frac{f_{i} \rho v_{i}^{2}}{2 D}\right) \Delta L
\end{aligned}
$$

O poço foi discretizado em segmentos (i) de comprimentos constantes para que as vazões mássicas pudessem ser obtidas individualmente para cada segmento, assim como, uma perda de carga associada a cada segmento, formando um perfil de pressão.

Conforme indicado na Equação 1, a vazão volumétrica acumulada de óleo $Q_{w i}$ é representada pela soma entre a vazão de óleo $Q_{R i}$ que está entrando no segmento i, output da simulação numérica, e a vazão de óleo acumulado até o segmento anterior a este $Q_{w-1}$.

Como o diâmetro do poço é conhecido, por meio da Equação 2, pode-se determinar a velocidade do óleo escoando em cada segmento i. Com as propriedades do óleo conhecidas e a velocidade, pode-se calcular o número de Reynolds para cada segmento i (Equação 3).

$\mathrm{Na}$ Equação 4, $f_{i}$ é o fator de atrito, $\mathrm{Re}_{\mathrm{i}}$ é o número de Reynolds (definido na Equação 3), $D$ é o diâmetro do poço e $\varepsilon$ é a rugosidade da parede do poço, definida, neste trabalho, como 1.E-4 m, para fins de comparação com trabalho de Ozkan et al. (1993). A correlação escolhida para o cálculo da perda de carga no interior do poço (Equação 4) foi a de Swamee-Jain (1976) válida para qualquer valor de número de Reynolds.

$\mathrm{Na}$ Equação 5, que é a clássica equação de Darcy-Weisbach, $\Delta P_{i}$ é a perda de carga no poço calculada para o i-ésimo segmento, $\rho$ é a massa específica do óleo, $L$ é o comprimento de cada segmento e $v_{i}$ é a velocidade do óleo referente à vazão acumulada no interior do poço para o i-ésimo segmento.

\section{RESULTADOS E DISCUSSÃO}

As simulações iniciais tiveram como objetivo validar a simulação numérica usando o software ANSYS FLUENT 13.0 com os dados de Ozkan et al. (1993), obtidos por meio de correlações. Desta forma, as mesmas condições daquele trabalho foram utilizadas como dados de entrada na simulação.

A Figura 3a apresenta os resultados da validação de dados entre o caso simulado e os dados de Ozkan et al. (1993) para a distribuição de vazão por unidade de comprimento ao longo do poço horizontal dada em $\mathrm{BPD} / \mathrm{m}$. A Figura $3 \mathrm{~b}$ apresenta os resultados em termos da produção acumulada ao longo do poço horizontal. 
De acordo com a Figura 3, os resultados da simulação numérica, para condutividade finita, concordam bem com os dados de Ozkan et al. (1993).

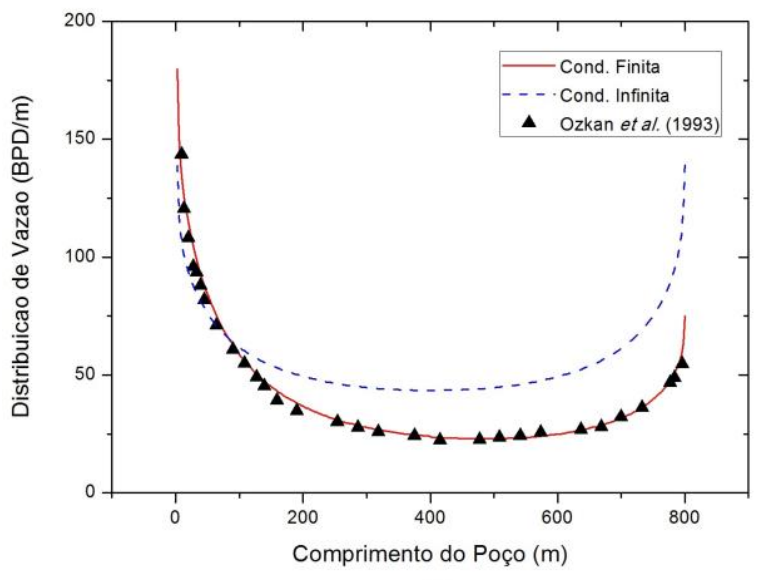

(a)

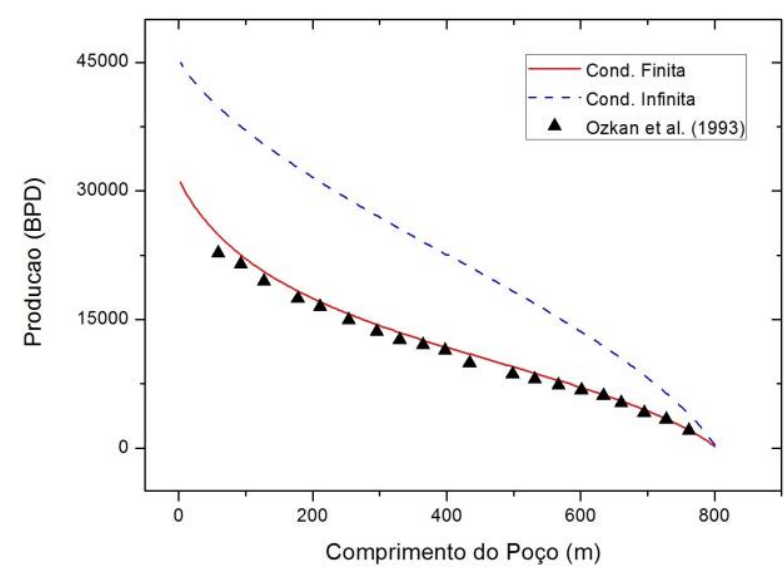

(b)

Figura 3 - Comparação (a) da distribuição de vazão e (b) da produção acumulada, ambos ao longo do poço, com os dados de Ozkan et al. (1993).

Como esperado, a região do toe $(800 \mathrm{~m})$ apresenta uma vazão por unidade de comprimento inferior se comparada com a mesma variável no heel $(0 \mathrm{~m})$, isto ocorre devido a orientação pela perda de carga em cada segmento originada pelo escoamento cumulativo na direção do próprio heel.

Entretanto, observa-se grande diferença quando o sistema é submetido à hipótese de condutividade infinita, como pode ser visto na Figura 3a. Neste caso, a distribuição de vazão adquire um caráter simétrico em relação às extremidades do poço. Isto se dá pela ausência de um perfil de pressão dominante.

Uma grande disparidade surge quando se analisa a simulação numérica sob efeito da condutividade infinita, porque a produção acumulada total (Figura 3b), verificada no heel, sofre um aumento significativo da ordem de $50 \%$, passando de 30.000 BPD para 45.000 BPD, quando comparada com a simulação com a condutividade finita. Isto revela que, para a formação de reservatório estudada, tal suposição acarretaria num superdimensionamento das previsões de produção.

A análise do drawdown (Figura 4) ao longo do poço revela uma queda de pressão da ordem de 12,54 psi compatível com a explicitada por meio das correlações de Ozkan et al. (1993), estimada em torno de 12,85 psi entre o toe e o heel, apresentando um erro relativo de cerca de $2,5 \%$.

Tendo em vista que a simulação numérica foi validada com os dados de Ozkan et al. (1993), pode-se variar parâmetros, como a rugosidade relativa e o comprimento do poço, para verificar o comportamento do sistema submetido a novas condições. 
A Figura 5a revela que, ao se elevar a rugosidade relativa, a curva de produção se desloca para baixo, o que representa uma redução na produtividade do poço. A explicação para tal comportamento está associada ao fato de que o fator de atrito (Equação 4) aumenta com o aumento da rugosidade, ocasionando uma perda de carga mais elevada, gerando, consequentemente, uma menor produção. Desta forma, para o sistema estudado, as melhores produções ocorrem para rugosidades relativas menores que $10^{-3}$. Para rugosidades menores que este valor, ocorre uma redução mais significativa da produção.

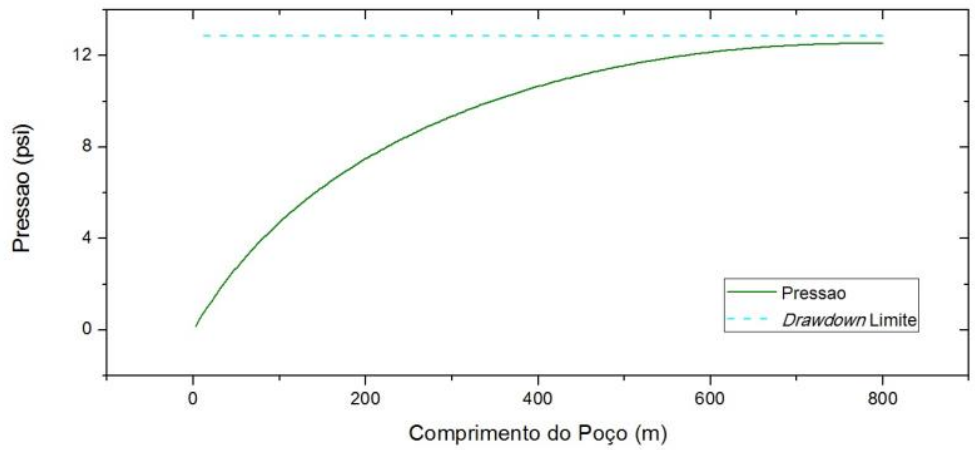

Figura 4 - Perfil de pressão do poço horizontal.

A Figura 5b apresenta a variação da produção acumulada quando o sistema foi submetido a diferentes comprimentos. É interessante notar que, para os maiores comprimentos (1.000 e $1.200 \mathrm{~m})$, o ganho de produtividade é relativamente baixo, pois para um incremento de 25 e $50 \%$ no comprimento, em relação a $800 \mathrm{~m}$, tem-se um aumento de pouco mais de $5 \%$ em BPD.

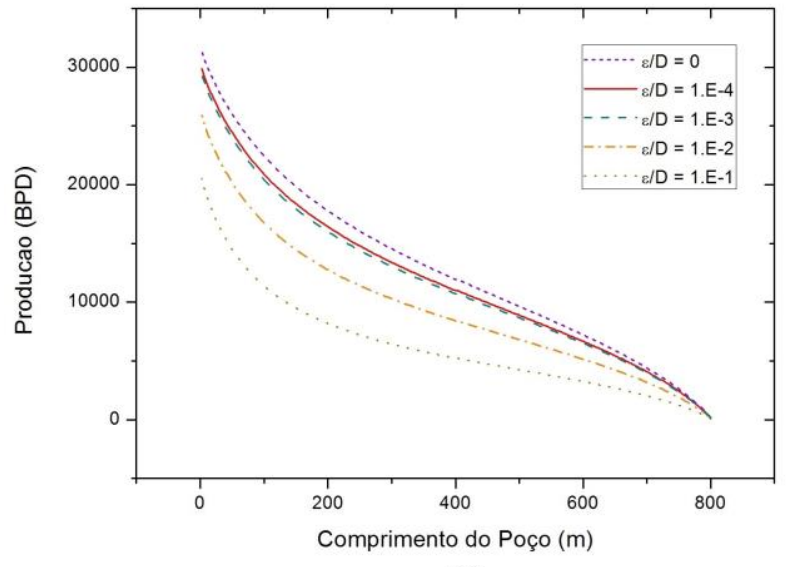

(a)

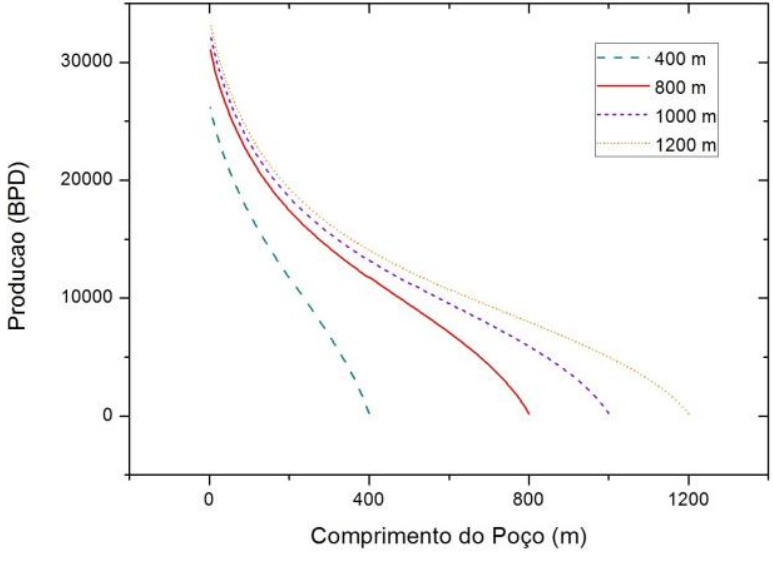

(b)

Figura 5 - Efeito (a) da rugosidade relativa e (b) do comprimento do poço na produção acumulada. 
Isto pode ser justificado pelo diferencial de pressão entre o reservatório e o poço que, para o sistema estudado, garante uma produção na faixa de 30.000 BPD para $800 \mathrm{~m}$ de poço. Qualquer incremento no comprimento do poço não é compensado em termos de produtividade, pois o drawdown, por se tratar de um parâmetro do campo, não acompanha esse incremento, e, portanto, não garante o escalonamento da produção. Em vista disso, comprimentos superiores a $800 \mathrm{~m}$ apresentam a desvantagem de encarecer o processo de exploração sem garantir um retorno em termos de produção.

\section{CONCLUSÃO}

O presente trabalho alcançou os objetivos inicialmente propostos, apresentando resultados coerentes com a modelagem e a física utilizada. A utilização do acoplamento poço-reservatório permitiu que os dados da simulação numérica fossem validados com os dados de Ozkan et al. (1993), evidenciando que a física do problema foi corretamente modelada.

No caso estudado, a distribuição de vazão sofre um deslocamento por conta da perda de carga em cada segmento do poço sob a condutividade finita, gerando uma menor vazão no toe que é compensada pelo aumento no heel. Isto explica a maior incidência de cones de água e gás na região do heel.

A influência da rugosidade relativa na produção é pequena para valores próximos de zero até $10^{-4}$, tornando-se relevantes para valores superiores a $10^{-3}$.

O comprimento do poço é uma variável importante na relação custo/benefício, uma vez que, para a situação analisada, comprimentos superiores a 800 m não compensam em termos econômicos, pois os ganhos de produção são pequenos.

\section{AGRADECIMENTOS}

Os autores agradecem ao CNPq e ao programa PRH-ANP/MCT, em particular ao PRH 13 da Escola de Química/UFRJ, pela concessão de uma bolsa de pós-doutorado e de uma bolsa de graduação, respectivamente.

\section{REFERÊNCIAS}

JOSHI, S.D. Horizontal Well Technology. PennWell Books, Tulsa, Oklahoma, USA, 1991.

OZKAN, E. SARICA, C. The Influence of Pressure Drop Along the Wellbore on Horizontal Well Productivity. SPE 25502, 1993.

SANSONI, U. FERNANDES, P.D. Study of oil reservoir coupling: the use of CFD tools for analysis of near well flow. Boletim Técnico da Produção de Petróleo: vol 2, n², p.287-302.Petrobras 2007. 